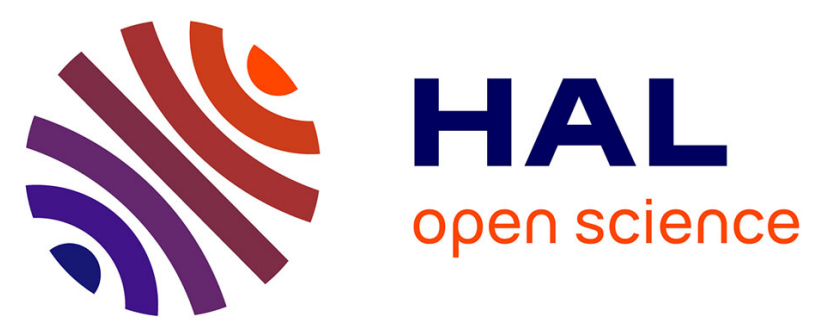

\title{
High aspect ratio taper-free micro and nano-channel fabrication in glass with ultrafast nondiffracting Bessel beams
}

F. Courvoisier, M.K. Bhuyan, M. Jacquot, P.-A. Lacourt, R. Salut, L. Furfaro, J.M. Dudley

\section{To cite this version:}

F. Courvoisier, M.K. Bhuyan, M. Jacquot, P.-A. Lacourt, R. Salut, et al.. High aspect ratio taperfree micro and nano-channel fabrication in glass with ultrafast nondiffracting Bessel beams. SPIEPhotonics West 2011, Jan 2011, San Francisco, United States. 10.1117/12.875409 hal-00661077

\section{HAL Id: hal-00661077 https://hal.science/hal-00661077}

Submitted on 15 Apr 2021

HAL is a multi-disciplinary open access archive for the deposit and dissemination of scientific research documents, whether they are published or not. The documents may come from teaching and research institutions in France or abroad, or from public or private research centers.
L'archive ouverte pluridisciplinaire HAL, est destinée au dépôt et à la diffusion de documents scientifiques de niveau recherche, publiés ou non, émanant des établissements d'enseignement et de recherche français ou étrangers, des laboratoires publics ou privés. 


\title{
High aspect ratio taper-free micro and nano-channel fabrication in glass with ultrafast nondiffracting Bessel beams
}

\author{
F. Courvoisier*, M.K. Bhuyan, M. Jacquot, P.A. Lacourt, R. Salut, L. Furfaro and John M. Dudley \\ FEMTO-ST Institute, Department of Optics P.M. Duffieux, UMR CNRS 6174, \\ Université de Franche-Comté, 25030 Besançon, France
}

\begin{abstract}
Ultrafast laser pulses are a powerful tool to process dielectrics. Here, we review our recent work concerning high aspect ratio micro and nanochannel processing in glass. We show how femtosecond Bessel beams overcome many of the difficulties associated with Gaussian beams. We report on single shot processing of nanochannels with aspect ratio up to 100. Underlying physical phenomena are discussed.
\end{abstract}

Keywords: Femtosecond laser, micromachining, high aspect ratio

\section{INTRODUCTION}

High aspect ratio laser micro/-nano processing is a challenge which aims at the fast realization of micro and nanofluidics structures or photonic components. For these applications, channels with diameters ranging between $50 \mathrm{~nm}$ and $500 \mathrm{~nm}$ over lengths exceeding several micrometers are required. For example, high quality photonic crystals need an aspect ratio (ie channel length over its diameter) over 10, that is not possible to achieve at present in materials such as glass or lithium niobate with focused ion beams techniques (FIB).

Laser processing with ultrafast pulses is a powerful technique that does not require vacuum and is capable of fast processing in 3D [1][2]. This technique is based on the control of extremely localized micro-explosions [3][4]. However, extrapolating this approach to the processing of long and thin channels is not straightforward.

Indeed, the nonlinear propagation of a gaussian beam modifies the beam structure through nonlinear Kerr effect, plasma generation, plasma absorption and defocusing. The electronic density of plasma channels produced by ultrashort gaussian beams or by aberrated gaussian beams therefore varies by several orders of magnitude along the optical axis. More, the technical control of such structures is difficult since the nonlinear processes involved render the pulse propagation extremely sensitive to the input conditions. Elongated voids and high aspect ratio channels have been demonstrated in the filamentary or geometrically aberrated propagation of gaussian beams, but many different regimes are observed, depending on the focusing conditions, energy per pulse and geometrical aberrations[5][6][7][8][9].

Here we show that nondiffracting Bessel beams allow to overcome many of the difficulties associated to Gaussian beams. We review studies of micro and nano-channel fabrication with femtosecond Bessel beams previously published in references [10], [11] and develop our interpretation of the physics of channel formation. First, we show that that taperfree micro-channels can be produced if the nonlinear propagation of Bessel beams corresponds to a stationary regime. In this framework, we secondly report the fabrication of nanochannels with diameters in the range 200-800 $\mathrm{nm}$ and aspect ratio up to $>100$. The underlying physical phenomena are then qualitatively discussed.

*francois.courvoisier@femto-st.fr, Phone +33 3816664 01, www.femto-st.fr 


\section{BESSEL BEAMS AND EXPERIMENTAL SETUP}

A Bessel beam is an axially symmetric interference of a set of plane waves whose wavevectors lie on a cone. Bessel beams were called "diffraction-free beam" by Durnin [12] since they correspond to a solution of the Helmoltz equation with an intensity profile invariant along the propagation direction. The intensity profile of a Bessel beam is composed by a central intense core surrounded by an infinite number of rings with lower intensity. In practice, Bessel beams are generated from input laser gaussian beams with an axicon lens or an optical system capable of applying a rotationally symmetric linear phase shift (Spatial Light Modulator, Fresnel zone plates, ring illumination in the focal plane of a lens $[13][14][15]$ etc).

Due to the finiteness of experimental numerical apertures, experimental Bessel beams obviously possess a finite number of surrounding lobes and exist only over a finite length. Bessel beams produced from Gaussian beams are described by Bessel-Gauss beams [16]. For shortness, we will abusively extend the name "Bessel beams" for Bessel-Gauss beams throughout this paper.

Finite-energy Bessel beams remain collimated over lengths that exceed the Rayleigh range by several orders of magnitude. In optics, this property has been used in the fields of optical manipulation [17], nonlinear harmonic generation [18], wave-packet localization [19], surface laser nanoprocessing [20] among others.

The experimental setup for Bessel beam generation is depicted on figure 1. The beam of a $100 \mathrm{fs} \mathrm{Ti}$ :Sa laser operating at $800 \mathrm{~nm}$ central wavelength is spatially shaped by reflection on a non-pixellized Spatial Light Modulator (SLM, Hamamatsu). The phase applied by the SLM phase mask reads as: $\Phi(\vec{r})=\beta\|\vec{r}\|+\overrightarrow{k_{1}} \cdot \vec{r} \quad[2 \pi]$

The first term is a rotationally symmetric linear phase that produces the Bessel beam. However, to separate different diffraction orders, the second linear term generates a grating in the direction of $k_{1}$. After spatial filtering in the focal plane of the first lens, the microscope objective performs a second Fourier transform that produces the Bessel beam starting at the focal plane. The setup is therefore equivalent to the generation of a virtual axicon at the focal plane of the microscope objective, thus preserving the working distance of the microscope objective. The virtual axicon can be generated within a transparent sample and is also transversally de-magnified by a factor $f_{1} l f_{2}$. Figure $1 \mathrm{~b}$ shows the experimental intensity map of a Bessel beam with $\beta=\pi \mathrm{mm}^{-1}, f_{l}=1 \mathrm{~m}, f_{2}=3.6 \mathrm{~mm}$. This corresponds to a Bessel beam of conical half-angle $\theta=24^{\circ}$ with respect to the optical axis. We note that the beam remain focused with a central core FWHM of $<0.7 \mu \mathrm{m}$ over a distance of $18 \mu \mathrm{m}$ in air.

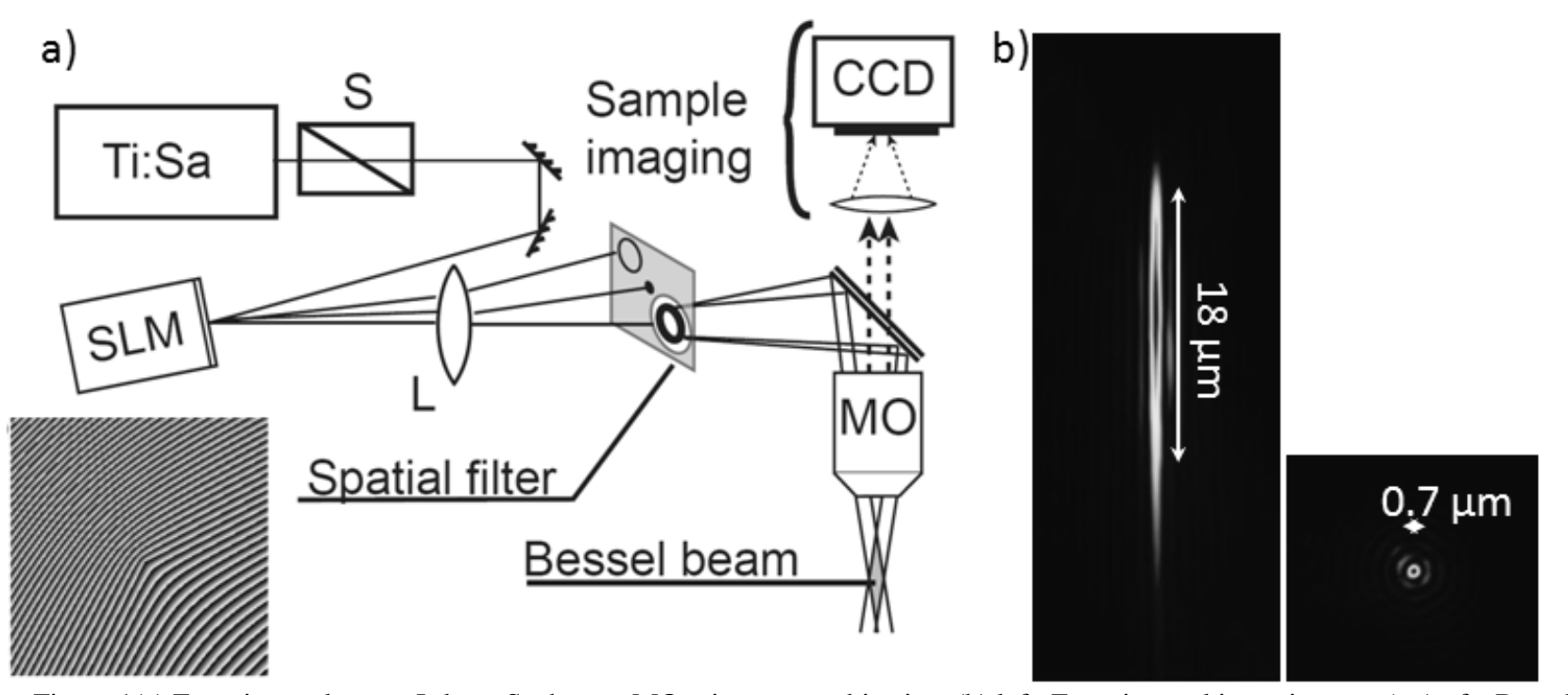

Figure 1(a) Experimental setup. L:lens, S: shutter, MO:microscope objective. (b) left: Experimental intensity map (r,z) of a Bessel beam with conical half angle $24^{\circ}$ in air (right) Intensity profile of the same beam. 


\section{WATER ASSISTED MICROCHANNEL PROCESSING IN THE MULTISHOT REGIME}

Bessel beams have only recently been used in systems driven by the nonlinear Schrödinger equation (NLSE) [21][22][23][24]. In the context of filamentation and nonlinear propagation in dielectrics, Bessel beams present a significant benefit to maintain intense laser-matter interaction over enhanced propagation lengths compared to gaussian beams. Indeed, for sufficiently high conical angle, the conical structure of Bessel beam allow to compensate the losses to due to multiphoton absorption and plasma generation thanks to an inward energy flow [23].This provides a way to obtain a wave experiencing nonlinear losses but propagating with a stationary intensity profile [25]. We also note this propagation regime behaves as an attractor to filamentation. Stationarity can be an important benefit for applications to high aspect ratio laser micro/nanostructuring.

In this section we review several results obtained in a systematic study reported in reference [10] concerning glass femtosecond laser processing in the multishot regime.

The setup used for this experiment is identical to the one reported in figure 1a, except that the microscope objective has a focal length of $9 \mathrm{~mm}$ (x20), thus producing a Bessel beam with conical half angle $10^{\circ}$ in glass, with a spot size of $1.5 \mu \mathrm{m}$ over more than $150 \mu \mathrm{m}$. The Bessel beam is focused at the exit side of a glass sample (Corning 0211). This sample side is in contact with water in order to remove debris out of the channel [26].

Figure $2 \mathrm{a}$ shows the focusing geometry as well as the channel produced after an illumination by $1000 \mathrm{laser}$ shots at $50 \mathrm{~Hz}$ and $7 \mu \mathrm{J}$ per pulse. The sample position is maintained fixed in the laser beam. The channel has an aspect ratio exceeding 40 and is taper-free. Figure $2 b$ shows the profile of a trench processed in the same experiment but where the sample is translated at a velocity of $0.5 \mathrm{~mm} / \mathrm{s}$ perpendicularly to the optical axis at $8 \mu \mathrm{J}$ per pulse. We attribute the parallelism of the trench and channel walls to the stationarity of the Bessel beam over the nonlinear propagation and plasma generation process.

Figure 2(c) shows the evolution of channel length and diameter with varying energy per pulse for a fixed 1000 laser shots. The error bars are related to the measurements on 10 different channels produced in the same conditions. After an onset regime close to the threshold for channel drilling, a stable region is observed. The energy range 7 to $8 \mu \mathrm{J}$ per pulse corresponds to a practical working window since the channels show a high regularity. A significant change is observed at $8 \mu \mathrm{J} /$ pulse. Indeed, from this energy, the channels present a high variability from channel to channel as well as in diameter.

a)

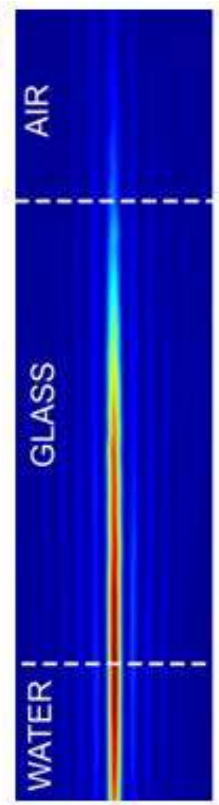

b)
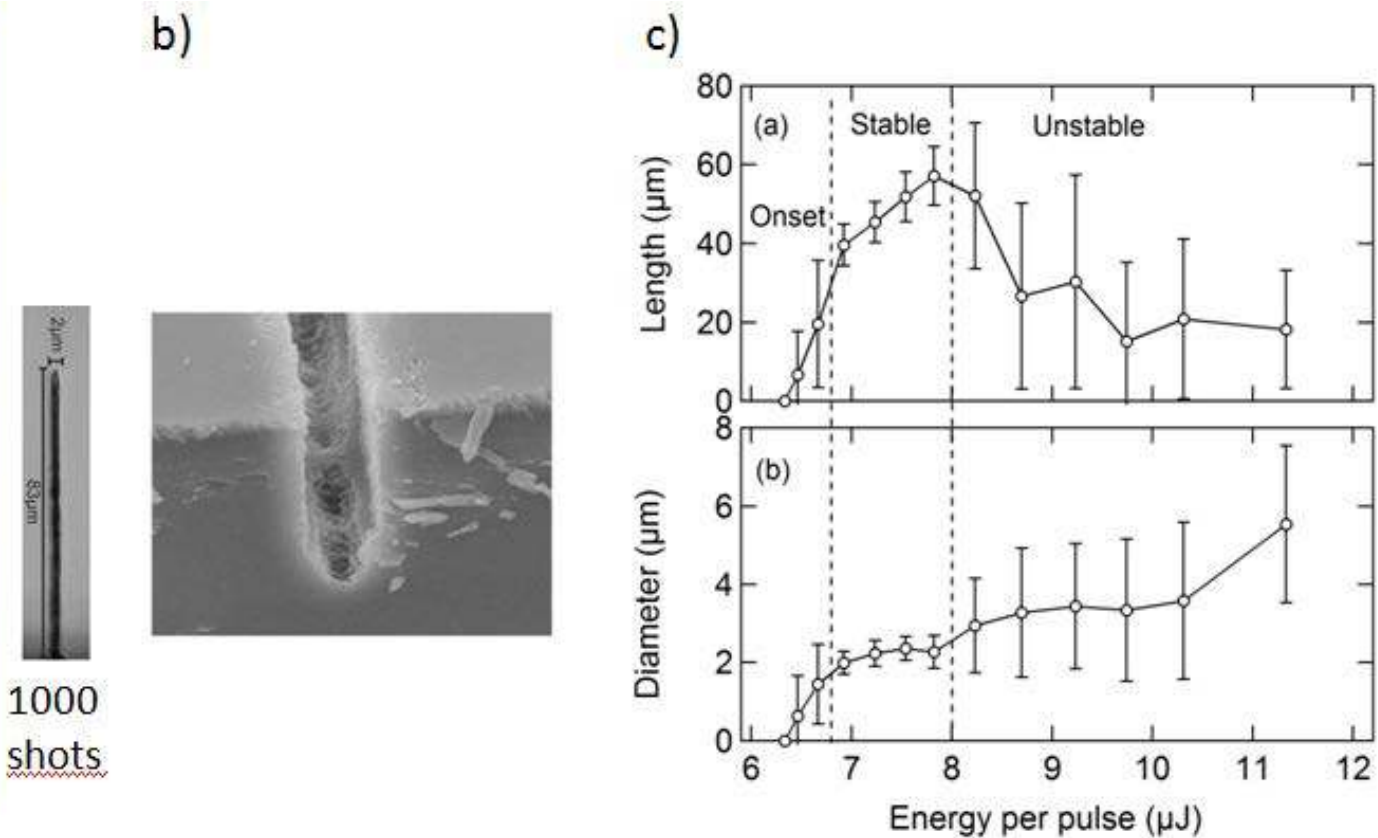

Figure 2 (a) Focusing geometry and channel produced without sample translation with 1000 laser shots at $50 \mathrm{~Hz}$ repetition rate and $7.6 \mu \mathrm{J} /$ pulse. (b) example of a trench produced by translating the sample perpendicularly to the beam with $5 \mathrm{kHz}$ repetition rate $(0.5 \mathrm{~mm} / \mathrm{s}, 8 \mu \mathrm{J} / \mathrm{pulse})$ (c) Evolution of channel length and diameter with energy per pulse $(50 \mathrm{~Hz}, 1000$ shots $)$ 
We interpret this threshold in the framework of Bessel filaments formation. Polesana et al [23] have shown that when a Bessel beam is formed within a dielectric sample, its propagation can be stationary provided sufficiently high power and high conical angle. They have also shown that under the same conditions, the propagation is no longer stationary if the air/dielectric is encountered by the beam when the Bessel beam is already formed. They interpret the de-stabilitization of the Bessel beam by the nonlinear index constrast at the air/dielectric interface. Similarly, our experiment can be interpreted in terms of nonlinear contrast at the interface, that also depends on the local intensity. Indeed, complimentary experiments have shows that reducing the intensity on the entrance side of the sample allow to maintain the stationarity over a larger energy per pulse window.

\section{SINGLE SHOT NANOCHANNEL PROCESSING}

In this section, we report a second set of experiments realized in single shot ablation regime, with high conical angles and a beam focused near the exit side in order to maintain stationarity. For this set of experiments, we used Bessel beams with two different conical half angles $\theta=11^{\circ}$ and $\theta=17^{\circ}$ in glass. The corresponding FWHM are $\sim 45$ and $30 \mu \mathrm{m}$ in the longitudinal direction and spot size $1.1 \mu \mathrm{m}$ and $0.7 \mu \mathrm{m}$ FWHM respectively.

Figure 3 shows the result of single shot ablation in four different experimental conditions. (a), (b) are performed for $\theta=17^{\circ}$ and pulse energy 0.65 and $0.85 \mu \mathrm{J}$ respectively. 3(c)(d) are performed with $\theta=11^{\circ}$ and pulse energy 1.9 and $2.1 \mu \mathrm{J}$. In all cases, a long nanochannel was formed. The aspect ratio of the structures ranges from 60 to 100 . We note that channels with so high aspect ratio are not achievable in dielectrics with other direct processing techniques. We also notice that in figure 3(a), several bridges are visible in the vicinity of the terminated end of the channel. We interpret these structures as being resolidified material. Indeed, at higher energies, these bridges are not visible and only nanoparticles are observed.

Figure 4 shows the evolution of channel length and diameter with energy for two different conical half angles. Below a threshold energy of $0.5 \mu \mathrm{J}$, only index modification is visible. Then, a transition regime takes place. In this transition regime, bridges are observed and the length of the channels increases rapidly. We note that the slope is higher for the more focused beam $\left(\theta=17^{\circ}\right)$. Then, the channel length evolves slowly with energy per pulse.

The channel length can be predicted with a simple intensity threshold model. Indeed, over the transition regime, channel length is fully in accordance with the distance between the point where the intensity of the central core first exceeds an intensity threshold of $5.10^{13} \mathrm{~W} / \mathrm{cm}^{2}$ and the exit side. This threshold intensity also corresponds to the intensity on the sample exit side at the minimal energy for channel formation $(0.5 \mu \mathrm{J})$. Figure $4(\mathrm{~b})$ shows the evolution of the central core intensity along the propagation direction for both conical half-angles. The solid lines in figure 4(top left) represent, for each energy, the length over which the intensity of the central core exceeds the threshold intensity.

Figure 4(left bottom) shows the evolution of channel diameter with energy. The solid lines show a linear fit of the data. We observe that the channel diameter evolves quasi-linearly with energy, and that it depends on energy density [11]. 




Figure 3 SEM images of cleaved nanochannels processed in glass with single laser shot: (a) $\theta=17^{\circ}, E=0.65 \mu \mathrm{J}$ (b) $\theta=17^{\circ}, \mathrm{E}=0.85 \mu \mathrm{J},(\mathrm{c}) \theta=11^{\circ}, \mathrm{E}=1.9 \mu \mathrm{J},(\mathrm{d}) \theta=11^{\circ}, \mathrm{E}=2.1 \mu \mathrm{J}$ 


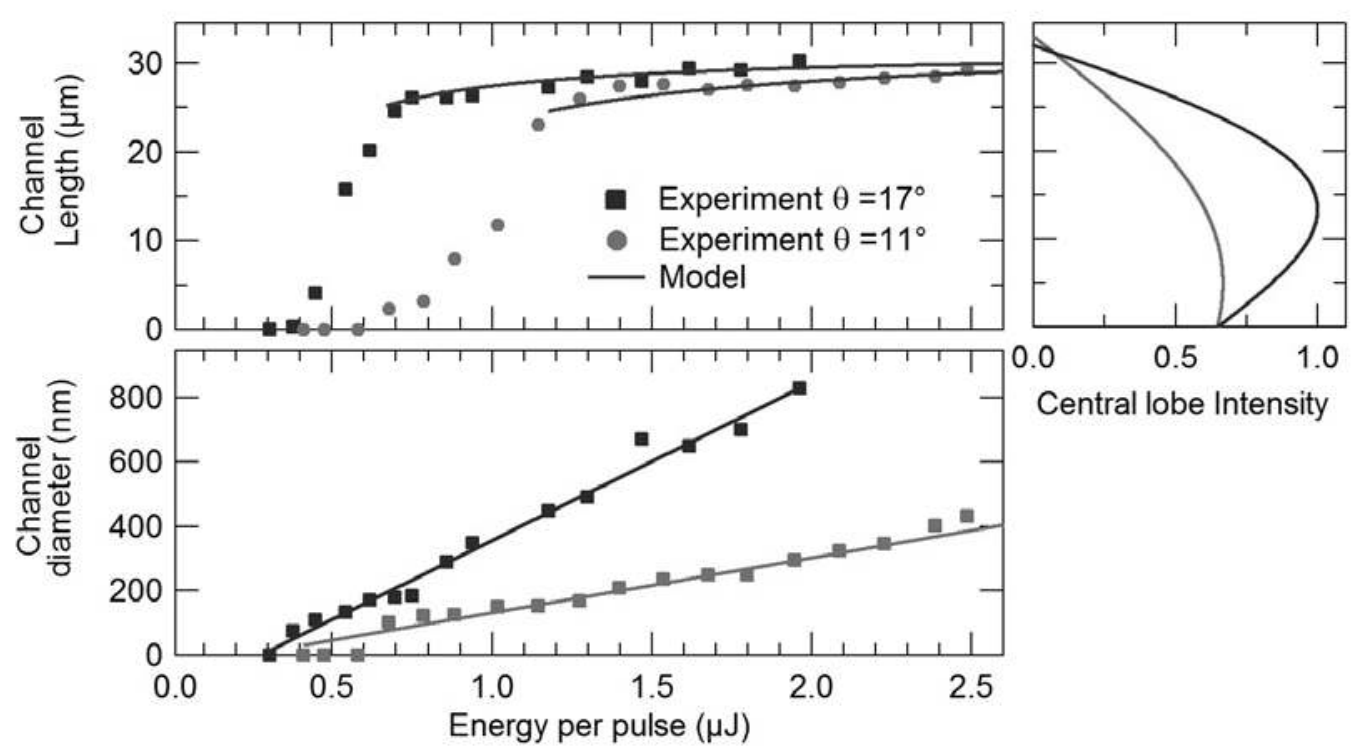

Figure 4 (left) Evolution of channel length and diameter with energy per pulse. Black squares correspond to $\theta=17^{\circ}$, grey circles correspond to $\theta=11^{\circ}$. The solid lines in the top panel correspond to the retrieved length according to an intensity threshold model. In the bottom panel, the straight lines correspond to linear fits of the channel diameter. (right) Evolution of the intensity in the central core with distance in the sample for $\theta=17^{\circ}$ (black) and $\theta=11^{\circ}$ (grey).

Figure 5 represents schematically our interpretation of the different physical processes leading to channel formation in the single shot regime, based on the micro-explosion model that was used to explain void formation in glass by tighly focused femtosecond pulses [3][4]. Although this interpretation still requires experimental and numerical confirmation, all results obtained throughout this study are compatible with this interpretation. In the first tens of femtosecond of the Bessel beam pulse, the intensity in the central core is high enough to trigger plasma formation. If the intensity of the central core is high enough, optical breakdown can be locally triggered. As explained before, the stationarity of Bessel beams does not change the beam profile, thus enabling the formation of a plasma channel with quasi-constant electronic density. During the relaxation of the plasma, the dielectric material is locally heated by the deposited energy. The quasiuniformity of the plasma channel density and temperature is transferred to the same quasi-uniform material heating. Then, thermal effects trigger phase changes, material expellation out of the channel, and material compression around the channel as well as resolidification for energies close to threshold.

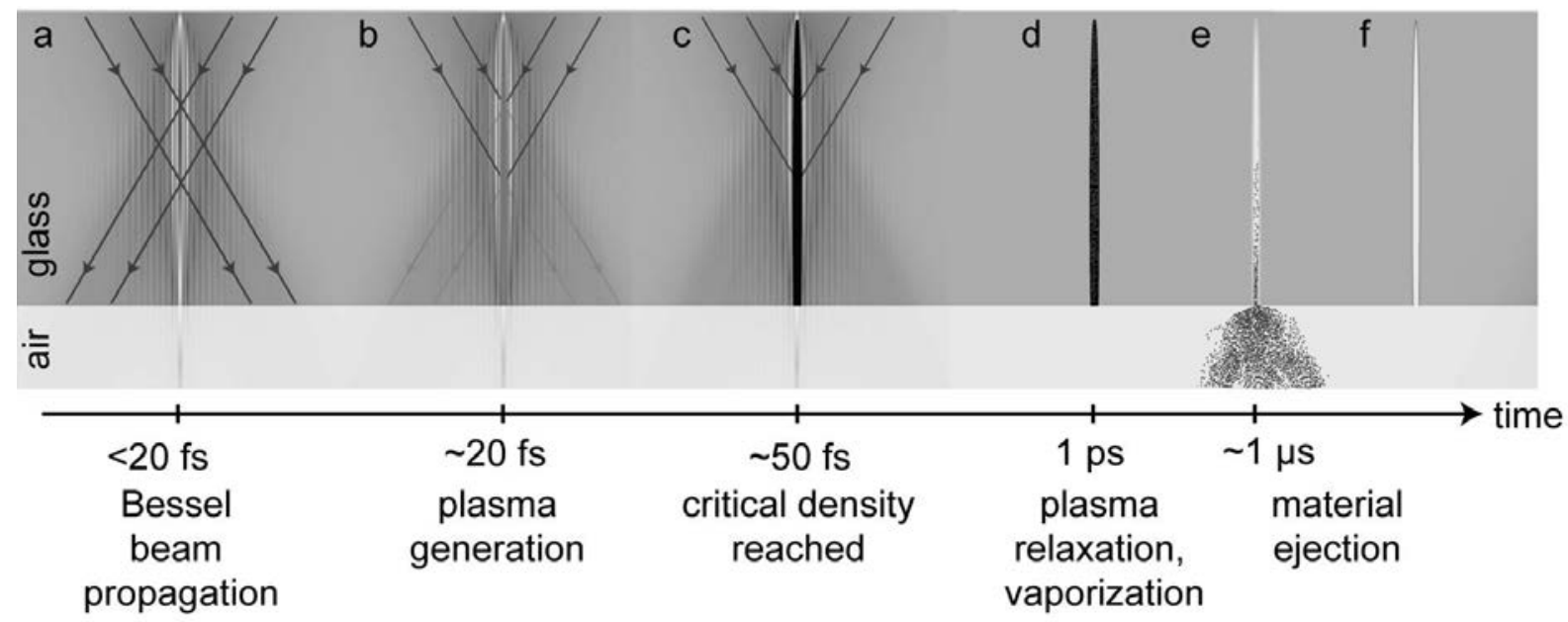

Figure 5 Interpretation of the physical phenomena yielding nanochannel formation. Given time values are typical numbers extracted from the literature. 
In this framework, channel length is determined by the length over which the intensity is higher than the intensity required to trigger optical breakdown. And the diameter obviously depends on local material heating i.e. on deposited energy density.

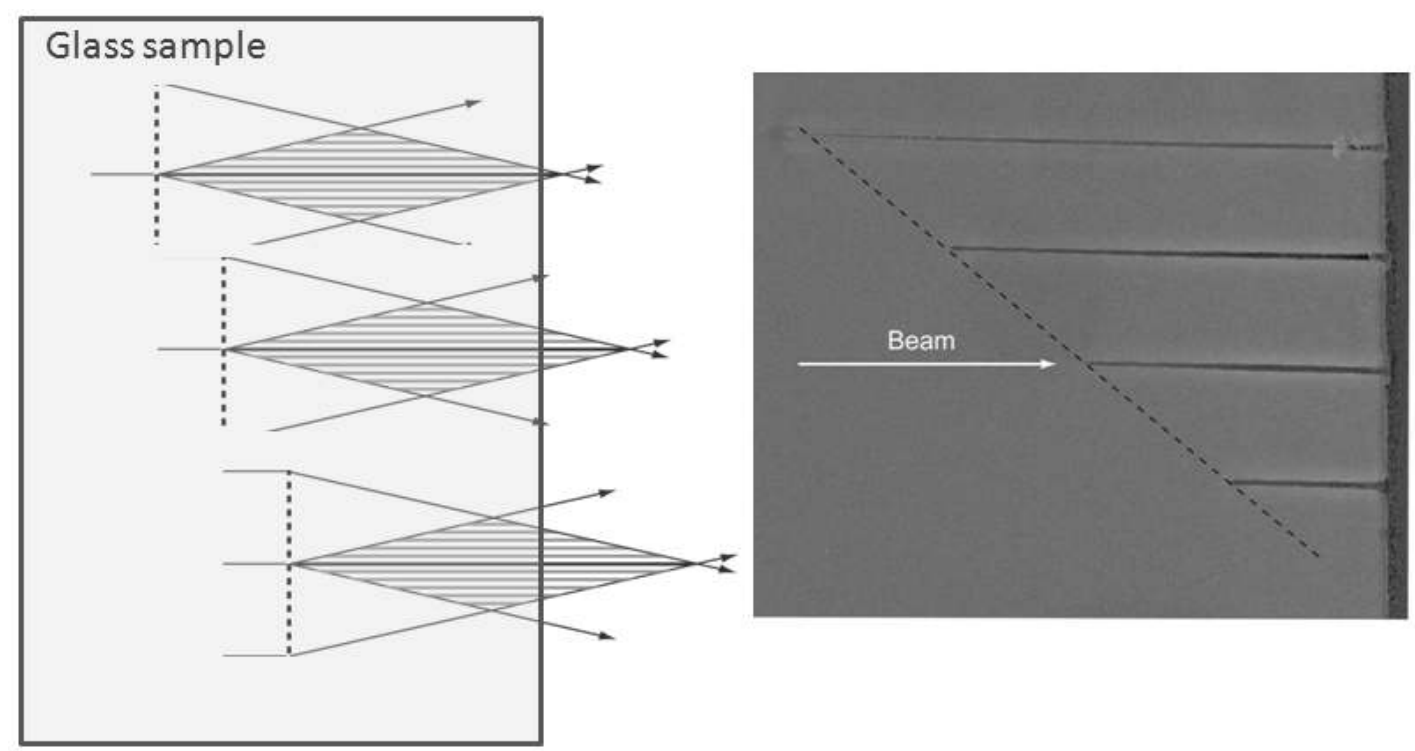

Figure 6 (left) experimental scheme (right) SEM image of the resulting structure (see text).

Figure 6 shows a SEM image of the profiles of channels produced by a sequence of four illuminations with identical pulse energies, but when the virtual axicon is linearly displaced by $4 \mu \mathrm{m}$ between each illumination. The dotted line shows the linearity of channel length. We also note that the diameter of the structures remains constant at $250 \mathrm{~nm}$. These observations are fully compatible with the model described previously.

This process can also be extended to process through-channels, though with reduced regularity [11]. The high repeatability of this process allows to generate periodic nanoctructures, as shown in reference [11].

The high repeatability and the linearity of the process with the input parameters demonstrate the capabilities of this novel laser nanoprocessing technique for further applications to nanofluidics and nanophotonics.

\section{CONCLUSION}

As conclusion, we have reviewed several results concerning micro-and nano-channel formation with ultrafast nondiffracting Bessel beams. The stationarity of Bessel beams at ablation level intensity was interpreted in the framework of Bessel filaments and is key for uniform energy deposition and high-aspect ratio channel formation. The linearity of the channel dimensions toward the input parameters is a significant benefit for technological applications.

\section{REFERENCES}

[1] Gattass, R.R. and Mazur, E., "Femtosecond laser micromachining in transparent materials," Nat. Photon. 2, 219-225 (2008).

[2] Gu, M., Jia, B., Li, J. and Ventura, M., "Fabrication of three-dimensional photonic crystals in quantum-dotbased materials," Laser Photonics Rev., 4, 414-431 (2010).

[3] Glezer, E.N. and Mazur, E., "Ultrafast-laser driven micro-explosions in transparent materials," Appl. Phys. Lett. 71, 882-884 (1997). 
[4] Juodkazis, S. et al., "Laser-Induced Microexplosion Confined in the Bulk of a Sapphire Crystal: Evidence of Multimegabar Pressures," Phys. Rev. Lett. 96, 166101 (2006).

[5] Couairon, A. and Mysyrowicz, A., "Femtosecond filamentation in transparent media," Phys. Rep. 441, 47-189 (2007).

[6] Nguyen, N.T., Saliminia, A., Liu, W., Chin, S.L. and Vallée, R. "Optical breakdown versus filamentation in fused silica by use of femtosecond infrared laser pulses," Opt. Lett. 28, 1591-1593 (2003).

[7] Kanehira, S., Si, J., Qiu, J., Fujita, K. and Hirao, K., "Periodic Nanovoid Structures via Femtosecond Laser Irradiation," Nano Letters 5, 1591-1595 (2005).

[8] White, Y.V., Li, X., Sikorski, Z., Davis, L.M. and Hofmeister, W., "Single-pulse ultrafast-laser machining of high aspect nano-holes at the surface of SiO2," Opt. Express 16, 14411-14420 (2008).

[9] Kudryashov, S.I., Mourou, G., Joglekar, A., Herbstman, J.F. and Hunt, A.J., "Nanochannels fabricated by highintensity femtosecond laser pulses on dielectric surfaces," Appl. Phys. Lett. 91, 141111-3 (2007).

[10] Bhuyan, M.K. et al, "High aspect ratio taper-free microchannel fabrication using femtosecond Bessel beams," Opt. Express 18, 566-574 (2010).

[11]Bhuyan, M.K. et al, "High aspect ratio nanochannel machining using single shot femtosecond Bessel beams," Appl. Phys. Lett. 97, 081102-081102-3 (2010).

[12] Durnin, J., Miceli, J.J. and Eberly, J.H., “Diffraction-free beams,” Phys. Rev. Lett. 58, 1499 (1987).

[13] Vasara, A., Turunen, J. and Friberg, A.T. "Realization of general nondiffracting beams with computergenerated holograms," J. Opt. Soc. Am. A 6, 1748-1754 (1989).

[14] McGloin, D. and Dholakia, K., " Bessel beams: diffraction in a new light," Contemp. Phys. 46, 15-28 (2005).

[15] Cizmar,T. and Dholakia, K., "Tunable Bessel light modes: engineering the axial propagation," Opt. Express 17, 15558-15570 (2009).

[16] Jarutis, V., Paskauskas, R. and Stabinis, A., "Focusing of Laguerre-Gaussian beams by axicon,” Opt. Commun. 184, 105-112 (2000).

[17] Garces-Chavez, V., McGloin, D., Melville, H., Sibbett, W. and Dholakia, K., "Simultaneous micromanipulation in multiple planes using a self-reconstructing light beam," Nature 419, 145-147 (2002).

[18] Van Dao, L., Dinh, K.B. and Hannaford, P. "Generation of extreme ultraviolet radiation with a BesselGaussian beam,” Appl. Phys. Lett. 95, 131114-3 (2009).

[19] Clerici, M. et al., "Space-time focusing of Bessel-like pulses," Opt. Lett. 35, 3267-3269 (2010).

[20] Courvoisier, F. et al. "Surface nanoprocessing with nondiffracting femtosecond Bessel beams," Opt. Lett. 34, 3163-3165 (2009).

[21] Polesana, P. et al., "High localization, focal depth and contrast by means of nonlinear Bessel beams," Opt. Express 13, 6160-6167 (2005).

[22] Polesana, P. et al. "Near-field dynamics of ultrashort pulsed Bessel beams in media with Kerr nonlinearity," Phys. Rev. E 73, 056612 (2006).

[23] Polesana, P., Franco, M., Couairon, A., Faccio, D. and Di Trapani, P. "Filamentation in Kerr media from pulsed Bessel beams," Phys. Rev. A 77, 043814 (2008).

[24] Polynkin, P. et al. "Generation of extended plasma channels in air using femtosecond Bessel beams," Opt. Express 16, 15733-15740 (2008).

[25] Porras, M.A., Parola, A., Faccio, D., Dubietis, A. and Trapani, P.D., "Nonlinear Unbalanced Bessel Beams: Stationary Conical Waves Supported by Nonlinear Losses," Phys. Rev. Lett. 93, 153902 (2004).

[26] Li, Y. et al., "Three-dimensional hole drilling of silica glass from the rear surface with femtosecond laser pulses," Opt. Lett. 26, 1912-1914 (2001). 